\title{
Cross-Unlinkable Hierarchical Group Signatures ${ }^{\star \star \star}$
}

\author{
Julien Bringer ${ }^{1}$, Hervé Chabanne ${ }^{12}$, and Alain Patey ${ }^{12}$ \\ 1 Morpho \\ 2 Télécom ParisTech \\ Identity \& Security Alliance (The Morpho and Télécom ParisTech Research Center)
}

\begin{abstract}
We introduce the notion of Cross-Unlinkability for group signature schemes. Considering groups organized in a tree structure, where belonging to the parent group is required to join a new group, CrossUnlinkability enables a cascade revocation process that takes into account the underlying tree structure, while ensuring anonymity for nonrevoked users, in particular, towards the managers of the other groups. We show how to achieve Cross-Unlinkability using the Verifier-Local Revocation group signature scheme of Bringer and Patey at Secrypt 2012, by exploiting its property of Backward Unlinkability.
\end{abstract}

Keywords: Anonymity, Unlinkability, Group Signatures

\section{Introduction}

Group signatures [9] enable authorized users to sign anonymously on behalf of a group. We consider in the following the case of VLR (Verifier-Local Revocation) group signatures. The VLR property [5] guarantees that only the public parameters and a revocation list RL are required to check a signature. Concretely, when a user is revoked, a revocation token that is derived from his signing key is added to RL. This token is used by verifiers to prevent revoked users from further signing.

In this paper we consider a scenario where users have access to several groups, equipped with group signatures, that have some dependencies between them: the set $\mathbb{G}$ of these groups is partially ordered and can be represented as a tree. When one wants to apply for new signing keys in a group $\mathcal{G}_{l}$, one has to own valid signing keys for the parent group $\mathcal{G}_{k}$ in the tree $\mathbb{G}$. This organization also requires that it should be possible to revoke automatically across different groups. To this aim, the new signing key is derived from the key of the same member for $\mathcal{G}_{k}$ in

* This work is partially funded under the European FP7 FIDELITY project (SEC2011-284862). All information is provided as is and no guarantee or warranty is given that the information is fit for any particular purpose. The European Commission has no liability in respect of this document, which merely represents the authors view.

** This is the extended version of the article presented at EuroPKI 2012. 
order to maintain a link. One important issue in our model is then to ensure the privacy of this link.

This scenario and the associated security properties are particularly adapted to identity management systems. In this setting, a user owns several identities derived from a strong identity (e.g. the national identity) while maintaining privacy and unlinkability between the different identities, even towards to the providers of the other identities.

We address this problem of derivation of group signatures keys from other group signature keys with privacy properties in mind. We in particular want to ensure that a given group manager cannot retrieve - except in case of revocations - the link between a signature in his group and a signature, issued by the same user, in any of his children groups. Our goal is to parallelize several instances of VLR group signatures while fulfilling the following additional requirements:

- A user registered into a given group should be able to sign anonymously on behalf of this group;

- When a user asks for registering to a new group, he has to prove that he can sign on behalf of the parent group, and the new keys delivered by the group manager should be derived from the pre-required keys;

- The derivation process should be compatible with a revocation process that echoes downwards, i.e. when a user $M_{i}$ is revoked from a given group $\mathcal{G}_{l}$, he must also be revoked from all the groups that are below $\mathcal{G}_{l}$ in the tree $\mathbb{G}$.

- Despite these revocation and derivation processes, only the manager of a given group $\mathcal{G}_{l}$ (and the signer) can learn information on the signer when looking at a signature for the group $\mathcal{G}_{l}$, provided this signer is not revoked from $\mathcal{G}_{l}$. Particularly, the other group managers learn nothing more than any observer and thus cannot link the signer to the members of their groups. This property, that we name Cross-Unlinkability, is an essential feature of our proposition.

Recall that, when a user is revoked, a revocation token that is derived from his signing key is added to RL. It enables to reject the further signatures of this user but it may also give a way to identify his previously made signatures. To prevent this, some VLR group signatures (e.g. $[8,15-17,19]$ ) enjoy an additional property called Backward Unlinkability (BU). The usual mechanism to enable BU is to split the time into several periods and derive revocation tokens associated to each period so that two revocation tokens for the same user for different periods are unlinkable.

We here adapt the derivation process for revocation tokens, no longer to enjoy BU, but to derive keys between the different group signature schemes. In our context defined above, the direction of the derivation is from a parent group $\mathcal{G}_{k}$ to a child group $\mathcal{G}_{l}$ in $\mathbb{G}$. In a sense, such a child group is seen as a time period for the VLR signature with BU associated to $\mathcal{G}_{k}$, unlinkability between different time periods in the original schemes with $\mathrm{BU}$ is transformed into unlinkability between the different children. We adapt the BU derivation process so that the new keys are not known by the parent group manager, while 
satisfying the requirement for the revocation process to be echoed to the lower levels.

For instance, consider the group tree described in Figure 1. We assume that a science faculty sets up a system using groups signatures, used for instance for access control. In this example, applying for a key for the Bioinformatics Team requires to previously own a key for the Computer Science Department. We also wish that, when one signs on behalf of, e.g., the Mechanics Department, anonymity of the signer is guaranteed against the managers of all other groups, including the managers of the parent group (Science Faculty), the children groups (Fluid Dynamics and Solid Mechanics) or the sibling groups (Computer Science Dept.).

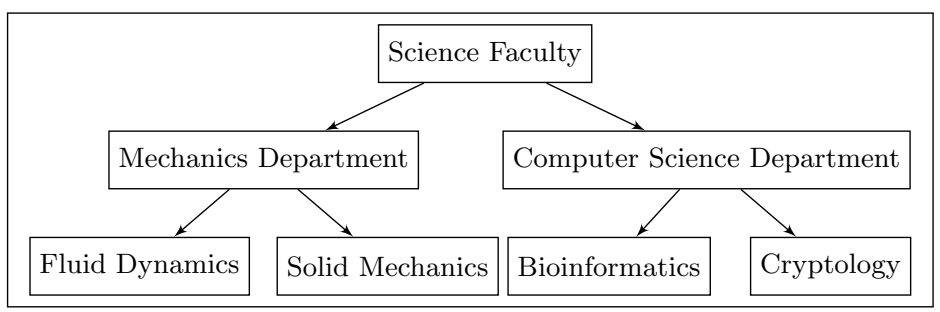

Fig. 1. An example of a group tree $\mathbb{G}$

Other settings with several parallel group signatures have already been introduced. Multi-group signatures $[1,3]$ enable a user to sign on behalf of either a single group or several groups to which he belongs. The notion of hierarchy between group signatures has been introduced in [14], where having a key for an upper group allows to sign on behalf of lower groups. Hierarchical Group Signatures [18] define a group organization that is close to ours: the managers are organized in a tree structure, but all of them do not manage signers, some only manage groups of managers; anonymity and (a weaker notion of) unlinkability between the users of different groups are considered but there is no possibility of revocation. Attribute-based group signatures [12,13], anonymous proxy signatures [11] and delegatable credentials [2] are also related notions. None of the above constructions however considers at the same time group hierarchy, unlinkability across the groups and revocation through the groups.

The process described in this paper is instantiated with the Bringer and Patey group signature [8] for a better readability but it can easily be adapted to other group signature schemes enjoying BU (e.g. [15-17, 19]).

In [6], Bringer et al. introduce the concept of cross-unlinkability for anonymous authentications, adapting the biometric-based remote authentication protocol of [7] to the hierarchical setting. In this paper, we formalize further this concept with the model of cross-unlinkable group signatures. We detail a con- 
struction and give its security properties. We finally prove that our construction satisfies these properties.

\section{VLR Group Signatures}

Group signatures [9] are a particular case of digital signatures where authorized members of a group are allowed to sign anonymously on behalf of the group. The anonymity can only be ended by the Group Manager who can also revoke misbehaving users (or users wanting to leave). In the particular case of VerifierLocal Revocation (VLR) [5], anyone knowing the public parameters of the group can verify the signatures (including revocation checks). Groups involved in VLR group signatures are dynamic: users can be revoked (voluntarily or not) at any time. The revocation process consists in adding revocation tokens to a public revocation list which is taken into account by the verifiers when they check a signature.

\subsection{Components}

The following algorithms are the components of a VLR group signature scheme with Backward Unlinkability. In the context of group signatures with BU, time is divided into time periods $j \in[1, \ldots, T]$. We denote by $\mathcal{G}$ the group associated to the group signature. If the signature does not enable BU, then the algorithms do not depend on time periods.

KeyGen $_{G S}$ : Generates the public parameters for the system and the public/secret keys of the group manager GM. It takes as input a security parameter $\mu$ and outputs the secret key $m s k$ of GM, its public counterpart $m p k$, an empty global revocation list $R L$ and empty period revocation lists $R L_{j}$ 's, for $j \in[1, \ldots, T]$, and the public parameters $g p k$. msk is kept secret by the GM, the other elements are published.

Join $_{G S}$ : Creates keys for a new user $M_{i}$ and allows him to produce group signatures. It outputs the user key $s k_{i}$ and the corresponding revocation tokens $r t_{i}$ (global revocation token) and $r t_{i j}$ (period), for all $j \in[1, \ldots, T] . s k_{i}$ is stored by the user and GM stores a part of $s k_{i}$ and revocation tokens.

$\operatorname{Sign}_{G S}$ : Takes as input a message $m$, a time period $j$ and a signer's key $s k_{i}$. Returns a signature $\sigma$ of user $M_{i}$ on the message $m$ at period $j$.

Verify $_{G S}$ : Takes as input a time period $j$, a message $m$, a signature $\sigma$ and the public parameters $g p k$ of the system and the current Revocation Lists $R L$ (global) and $R L_{j}$ (period). Checks if the message has been well-signed by an unrevoked group member without revealing the signer's identity.

Open $_{G S}$ : Takes a signature $\sigma$ as input and reveals the identity of the signer. Can only be performed by the GM, since it requires to know the revocation tokens of all users.

Revoke $_{G S}$ : Revokes a user $M_{i}$ from the group at a time period $j$. His revocation token $r t_{i j}$ is added to $R L_{j}$. (For a global revocation, the same process is executed with $r t_{i}$ and $R L$.) 


\subsection{Security properties}

We describe the security properties that can be required from a VLR group signature scheme with BU. Our description is a slight variant of the one of [8] but it is also fulfilled by the [8] scheme. In particular, we do not require the games to follow a chronological order, since it is not necessary in the proofs of security of $[8]$.

Correctness: Every check of a well-formed signature returns valid if the user who has issued it is not revoked.

The Traceability property ensures that no attacker (or group of attackers) is able to forge a signature that can not be traced to one of the corrupted users which participated in its forgery.

Traceability: Let us consider the following Traceability game played by an adversary $\mathbf{A}$.

Setup: The challenger $\mathbf{C}$ runs the KeyGen algorithm, playing the role of the GM. He obtains $g p k, m p k$ and $m s k$. He provides $\mathbf{A}$ with $g p k$ and $m p k$. Queries: A can make the following queries to the challenger, provided that it well specifies the time period $j$ :

- Join: A requests the enrolment of a member $M_{i}$ to $\mathcal{G}$. $\mathbf{C}$ obtains the keys for $M_{i}$ for $\mathcal{G}$. A obtains nothing.

- Sign: A requests that a member $M_{i}$ of $\mathcal{G}$ signs a message $m$ for the current period $j$. The challenger computes the signature $\sigma$ of $M_{i}$ on $m$ for $j$. A obtains $\sigma$.

- Corruption: A requests the corruption of a given registered member $M_{i}$. He obtains the secret key of $M_{i}$ for $\mathcal{G}$. The member $M_{i}$ is revoked at all time periods.

- Revocation: A requests the revocation of a user $M_{i}$ from $\mathcal{G}$ for period $j$. He learns the revocation token of $M_{i}$ that is disclosed during this phase. Output: A outputs a challenge message $m$, a period $j$ and a signature $\sigma$ on $m$ and wins if:

1. $\operatorname{Verify}_{G S}\left(m, \sigma, j, g p k, m p k, R L_{j}\right)=$ valid

2. A did not obtain $\sigma$ by making a Sign Query on $m$.

The scheme is said to satisfy Traceability if no polynomial probabilistic adversary is able to win the above game with a non-negligible probability.

The Backward Unlinkability property is an extension of the notion of SelflessAnonymity [5] to the multi-period setting. Selfless-Anonymity implies that only the signer and the Group Manager learn information on the producer of a given signature, provided that the signer is not revoked. Backward Unlinkability moreover ensures that valid signatures remain anonymous even after the signer's revocation. Revoked users can also come back after their revocation into the group and use their previous keys without any loss of anonymity. That is why we require time to be divided into several periods so that the signer uses different parameters for the different periods. 
However notice that when one is revoked at a given period, he loses anonymity on all his signatures issued at this particular period, even if they were produced before the revocation. Anonymity is only guaranteed on the signatures made at previous periods where the user was not revoked.

The Selfless-Anonymity definition can be obtained from the definition of Backward Unlinkability by applying it to the case where only one time period is available.

Backward Unlinkabilty: Let us consider the following BU game played by an adversary A:

Setup: The challenger $\mathbf{C}$ runs the KeyGen algorithm, playing the role of the GM. He obtains $g p k, m p k$ and $m s k$. He provides A with $g p k$ and $m p k$. Queries: A can make the following queries to the challenger, provided that it well specifies the time period $j$ :

- Join: A requests the enrolment of a member $M_{i}$ to $\mathcal{G}$. $\mathbf{C}$ obtains the keys of $M_{i}$ for $\mathcal{G}$. A obtains nothing.

- Sign: A requests that a member $M_{i}$ of $\mathcal{G}$ signs a message $m$ for the current period $j$. The challenger computes the signature $\sigma$ of $M_{i}$ on $m$ for $j$. A obtains $\sigma$.

- Corruption: A requests the corruption of a given registered member $M_{i}$. He obtains the secret key of $M_{i}$ for $\mathcal{G}$.

- Revocation: A requests the revocation of a user $M_{i}$ from $\mathcal{G}$ for period $j$. He learns the revocation token of $M_{i}$ that is disclosed during this phase.

Challenge: A outputs a challenge message $m$, a period $j$ and two different members $M_{0}$ and $M_{1}$, such that:

1. $M_{0}$ and $M_{1}$ are both registered to $\mathcal{G}$;

2. A corrupted neither $M_{0}$ nor $M_{1}$;

3. $M_{0}$ and $M_{1}$ are not revoked from $\mathcal{G}$ at period $j$

$\mathbf{C}$ chooses a random bits $b \in_{R}\{0,1\}$ and runs $\operatorname{Sign}_{G S}$ for $M_{b}$ at period $j$ using message $m$. The obtained signature $\sigma^{*}$ is transmitted to $\mathbf{A}$.

Restricted Queries: A can make the same queries as in the Queries phase, as long as this does not contradict the above requirements 1 to 3 of the Challenge phase.

Output: A outputs a guess $\beta \in\{0,1\}$ on $b$.

The scheme satisfies Backward-Unlinkability if the probability $\mid \operatorname{Pr}(\beta=b)-$ $1 / 2 \mid$ is negligible.

Exculpability Nobody, even the Group Manager, is able to produce another user's signature.

(This property is not always satisfied by VLR group signature schemes. We refer the reader to $[8,10]$ for a formal definition that is adapted to the schemes used in the following.)

\subsection{The CL and BP Schemes}

As an example and for a better understanding, we use the scheme of Bringer and Patey [8], that we denote by BP, that fulfils all the above security requirements. Particularly, it enables Backward Unlinkability using the usual technique 
of dividing the time into several periods and deriving revocation tokens for the members that depend on the time and on the secret key of the user but that cannot be linked with each other. We moreover use the patched version of the scheme of Chen and $\mathrm{Li}[10]$, also described in [8], that we denote by CL, and that is merely the BP scheme without BU. In particular, we can use the same parameters and keys for both schemes.

We first describe the CL scheme. Notice that, since it does not enable BU, algorithms are independent of the time period.

$\operatorname{KeyGen}_{C L}(\mu)$ Choose bilinear groups $G_{1}, G_{2}, G_{\mathcal{T}}$ of order a $\mu$-bit prime number $p$ that is safe, a prime number $q$ and a pairing $e: G_{1} \times G_{2} \rightarrow G_{\mathcal{T}}$. Let $g_{1}, g_{2}$ be generators of $G_{1}$ and $G_{2}$. Choose a hash function $H:\{0,1\}^{*} \rightarrow \mathbb{Z}_{p}$ . Choose $\tilde{g}_{1}, \hat{g}_{1} \in_{R} G_{1}, \gamma \in \in_{R} \mathbb{Z}_{p}^{*}$, and compute $w=g_{2}^{\gamma}$. Compute $T_{1}=$ $e\left(g_{1}, g_{2}\right), T_{2}=e\left(\tilde{g}_{1}, g_{2}\right), T_{3}=e\left(\hat{g}_{1}, g_{2}\right)$ and $T_{4}=e\left(\hat{g}_{1}, w\right)$. Output: $g p k=$ $\left(G_{1}, G_{2}, G_{\mathcal{T}}, e, p, g_{1}, g_{2}, \tilde{g}_{1}, \hat{g}_{1}, w, H, T_{1}, T_{2}, T_{3}, T_{4}\right.$ and $m s k=\gamma$.

$\operatorname{Join}_{C L}\left(M_{i}, m s k, g p k, m p k\right) \mathrm{GM}$ sends a nonce $n_{i} \in\{0,1\}^{k}$ to $M_{i} . M_{i}$ chooses $f_{i} \in R \mathbb{Z}_{p}$ and computes $F_{i}=\tilde{g}_{1}^{f_{i}}$. He chooses $r_{f} \in \in_{R} \mathbb{Z}_{p}$ and computes $R=\tilde{g}_{1}^{r_{f}}$. He computes $c=H\left(g p k\left\|F_{i}\right\| R \| n_{i}\right)$ then $s_{f}=r_{f}+c f_{i} . M_{i}$ sends comm $=\left(F_{i}, c, s_{f}\right)$ to GM. GM computes $R^{\prime}=\tilde{g}_{1}^{s_{f}} F_{i}^{-c}$ and checks that $s_{f} \in \mathbb{Z}_{p}$ and $c=H\left(g p k\|F\| R^{\prime} \| n_{i}\right)$. He chooses $x_{i} \in \mathbb{Z}_{p}$ and computes $A_{i}=$ $\left(g_{1} F_{i}\right)^{1 /\left(x_{i}+\gamma\right)}$. GM sends $\left(A_{i}, x_{i}\right)$ to $M_{i}$, using a secure channel. $M_{i}$ checks that $e\left(A_{i}, w g_{2}^{x_{i}}\right)=e\left(g_{1} \tilde{g}_{1}^{f_{i}}, g_{2}\right)$ and outputs $s k_{i}=\left(f_{i}, x_{i}, A_{i}\right)$. The global revocation token for $M_{i}$ is $r t_{i}=x_{i}$. GM outputs $x_{i}, A_{i}$ and the revocation tokens.

$\operatorname{Sign}_{C L}\left(m, s k_{i}, g p k, m p k\right)$ Choose $B \in_{R} G_{1}$ and compute $J=B^{f_{i}}, K=B^{x_{i}}$. Choose $a \in_{R} \mathbb{Z}_{p}$, compute $b=a x_{i}$ and $T=A_{i} \hat{g}_{1}^{a}$. Choose $r_{f}, r_{x}, r_{a}, r_{b} \in \in_{R} \mathbb{Z}_{p}$. Compute $R_{1}=B^{r_{f}}, R_{2}=B^{r_{x}}, R_{4}=K^{r_{a}} B^{-r_{b}}, R_{3}=e\left(T, g_{2}\right)^{-r_{x}} T_{2}^{r_{f}} T_{3}^{r_{b}} T_{4}^{r_{a}}$. Compute $c=H\left(g p k|| B|| J|| K|| T|| R_{1}|| R_{2}|| R_{3}|| R_{4}|| m\right)$. Compute $s_{f}=r_{f}+$ $c f_{i}, s_{x}=r_{x}+c x_{i}, s_{a}=r_{a}+c a$ and $s_{b}=r_{b}+c b$. Output: $\sigma=\left(B, J, K, T, c, s_{f}\right.$, $\left.s_{x}, s_{a}, s_{b}, \mathbf{s}_{\mathbf{1}}, \ldots, \mathbf{s}_{\lambda}\right)$.

$\operatorname{Verify}_{C L}(m, \sigma, g p k, m p k, R L)$ 1. Signature Check:

Check that $B, J, K, T \in G_{1}$ and $s_{f}, s_{x}, s_{a}, s_{b}, \mathbf{s}_{\mathbf{1}}, \ldots, \mathbf{s}_{\lambda} \in \mathbb{Z}_{p}$. Compute $R_{1}^{\prime}=B^{s_{f}} J^{-c}, R_{2}^{\prime}=B^{s_{x}} K^{-c}, R_{3}^{\prime}=e\left(T, g_{2}\right)^{-s_{x}} T_{2}^{s_{f}} T_{3}^{s_{b}} T_{4}^{s_{a}} T_{1}^{c} e(T, w)^{-c}$ and $R_{4}^{\prime}=K^{s_{a}} B^{-s_{b}}$. Check that $c=H\left(g p k\|B\| J\|K\| T\left\|R_{1}^{\prime}\right\| R_{2}^{\prime}|| R_{3}^{\prime} \| R_{4}^{\prime} \mid\right.$ $\mid m)$.

2. Revocation Check: Check that $\forall r t_{i} \in R L, K \neq B^{r t_{i}}$. Output valid if all checks succeed. Otherwise output invalid.

Revoke $_{C L}\left(R L, r t_{i}\right)$ Add the member's revocation token $r t_{i}$ to the current revocation list $R L$ and publish the thus updated $R L$.

Open $_{C L}\left(\sigma, g p k, m p k, m s k,\left\{r t_{i} \mid M_{i}\right.\right.$ is in the group $\left.\}\right)$ For every member $M_{i} \in$ $\mathcal{G}$, use the Revocation Check algorithm on the signature $\sigma$ with a revocation list set as $R L=\left\{r t_{i}\right\}$. When the test fails, output the corresponding $M_{i}$.

We now describe the BP scheme. Notice that, since it enables BU, algorithms depend on the time period. 
$\operatorname{KeyGen}_{B P}(\mu)$ Run $\operatorname{KeyGen}_{C L}(\mu)$. Furthermore, choose a security parameter $\lambda$ for the proofs of knowledge involving double logarithms. Pick $h_{1}, \ldots, h_{T} \in_{R}$ $\mathbb{Z}_{q}^{*}$ and add $\lambda$ and the $h_{j}$ 's to $g p k$

$\operatorname{Join}_{B P}\left(M_{i}, m s k, g p k, m p k\right)$ Run $\mathbf{J o i n}_{C L}\left(M_{i}, m s k, g p k, m p k\right)$. Moreover, the revocation token for $M_{i}$ at period $j$ is $r t_{i j}=h_{j}^{x_{i}}$.

$\operatorname{Sign}_{B P}\left(m, j, s k_{i}, g p k, m p k\right)$ Run the $\operatorname{Sign}_{C L}\left(m, s k_{i}, g p k, m p k\right)$ algorithm with some adaptations: in addition to $B, J$ and $K$, compute $L=B^{h_{j}^{x_{i}}}$ and add $j$ and $L$ in the input of the hash function to compute $c$. Moreover, pick $r_{1}, \ldots, r_{\lambda} \in_{R} \mathbb{Z}_{p}$. Compute $V_{l}=B^{r_{l}}$ and $W_{l}=B^{h_{j}^{r_{l}}}, \forall l=1 \ldots \lambda$. Compute $d=H\left(c \|\left(V_{l}, W_{l}\right)_{l=1 \ldots \lambda}\right) . \forall l=1 \ldots \lambda$, let $b_{l}$ be the $l^{\text {th }}$ bit of $d$. Set $s_{l}=r_{l}-b_{l} x$. Add $L, d, s_{1}, \ldots, s_{\lambda}$ to the output.

$\operatorname{Verify}_{B P}\left(m, \sigma, j, g p k, m p k, R L, R L_{j}\right) \quad$ 1. Signature Check:

Run the Signature Check of $\operatorname{Verify}_{C L}(m, \sigma, g p k, m p k, R L)$ with some adaptations: check that $L \in G_{1}$ and that $s_{1}, \ldots, s_{\lambda} \in \mathbb{Z}_{p}$, and add $j$ and $L$ in the input of the hash function to compute $c . \forall l=1 \ldots \lambda$, let $b_{l}$ be the $l^{\text {th }}$ bit of $d$.. Compute $V_{l}^{\prime}=B^{s_{l}} K^{b_{l}}$ and $W_{l}^{\prime}=\left(B^{1-b_{l}} L^{b_{l}}\right)^{h_{j}^{s_{l}}}$. Check that $d=H\left(c^{\prime} \|\left(V_{l}^{\prime}, W_{l}^{\prime}\right)_{l=1 \ldots \lambda}\right)$.

2. Revocation Check:

Run the Revocation Check of Verify ${ }_{C L}(m, \sigma, g p k, m p k, R L)$. Moreover, check that $\forall r t_{i j} \in R L_{j}, L \neq B^{r t_{i j}}$. Output valid if all checks succeed. Otherwise output invalid.

Revoke $_{B P}\left(j, R L, R L_{j}, r t_{i}, r t_{i j}\right)$ For a global revocation, run $\operatorname{Revoke}_{C L}\left(R L, r t_{i}\right)$. For a period revocation, add the member's revocation token $r t_{i j}$ to the current revocation list $R L_{j}$ and publish the thus updated $R L_{j}$.

$\operatorname{Open}_{B P}\left(\sigma, j, g p k, m p k, m s k,\left\{r t_{i j} \mid M_{i}\right.\right.$ is in the group $\left.\}\right)$ For every member $M_{i}$ $\in \mathcal{G}$, use the Revocation Check algorithm on the signature $\sigma$ with a revocation list set as $R L_{j}=\left\{r t_{i j}\right\}$. When the test fails, output the corresponding $M_{i}$.

Notice that the $\mathbf{R e v o k e}_{G S}$ and $\mathbf{O p e n}_{G S}$ are standard procedures of VLR group signature schemes and are not specific to the BP and CL schemes.

We recall the security results given in $[8,10]$, where DDH refers to the Decisional Diffie-Hellman assumption, DL to the Discrete Logarithm assumption and $q$-SDH refers to the $q$-Strong Diffie-Hellman assumption [4]. The adapted $\mathrm{DHH}$ is an adaptation of the DDH assumption described in [8].

\section{Theorem 1 (Security of the BP and CL Schemes).}

In the random oracle model, the CL scheme described above achieves Correctness, Selfless Anonymity (under the DDH assumption), Exculpability (under the DL assumption) and Traceability (under the q-SDH assumption).

In the random oracle model, the BP scheme described above achieves Correctness, Backward Unlinkability (under the adapted DDH assumption), Exculpability (under the DL assumption) and Traceability (under the q-SDH assumption). 


\section{Our Model of Cross-Unlinkable Hierarchical Group Signatures}

We here describe our model for a hierarchical group signature setting where groups follow a tree hierarchy. Our goal is to constrain that, when one wants to acquire a group signature key for a group $\mathcal{G}_{k}$, one has to prove that one belongs to the parent group in the tree $\mathbb{G}$. Our model does not change much the way the members of a particular group use group signatures. It focuses on the way the member keys for the different groups are linked to enable at the same time a cascade derivation process and unlinkability between signatures issued by the same user for different groups, in particular towards the group managers. In our model, only the signer and the group manager of the concerned group (and particularly not the other GM's) are able to identify the producer of a given signature. We also precise that, within a group, the way we use signatures does not enable us to enjoy BU. Nevertheless, we achieve Selfless-Anonymity [5] as for usual VLR group signature schemes, where, still, only the signer and the GM are able to tell who produced a particular signature but where, once a member has been revoked, he loses his anonymity on all his signatures.

\subsection{Setting}

We assume that there are several groups $\mathcal{G}_{k}$ organized as a tree $\mathbb{G}$ with a root $\mathcal{G}_{0}$. Each group $\mathcal{G}_{l}$ has a group manager $G M_{l}$ and we will denote by $k \dashv l$ the fact that the group $\mathcal{G}_{k}$ is a parent of the group $\mathcal{G}_{l}$. The functionalities of our protocol are the following.

KeyGen is an extension of the $\mathbf{K e y G e n}_{G S}$ algorithm to the hierarchical group setting, it specifies how the parameter choices of the different group managers should be related.

$\operatorname{KeyGen}(\lambda)$ : This is run by the GM's. It takes as input a security parameter

$\lambda . G M_{0}$ first returns the public parameters $g p k$ for all the group signatures. Then each $G M_{l}$ creates a secret/public key pair $\left(m s k^{l}, m p k^{l}\right)$ and publishes $m p k^{l}$.

The Enrolment algorithm specifies how a group manager $G M_{l}$ and a user $M_{i}$ applying to join $\mathcal{G}_{l}$ interact to provide $M_{i}$ with a key for $\mathcal{G}_{l}$. If $\mathcal{G}_{l} \neq \mathcal{G}_{0}$, this algorithm calls the Derivation algorithm, that we describe next.

$\operatorname{Enrolment}\left(M_{i}, \mathcal{G}_{l}\right)$ : For a group $\mathcal{G}_{l}$, this algorithm is jointly run by the group manager $G M_{l}$ and a user $M_{i}$. The input for $G M_{l}$ is his secret key $m s k^{l}$, it also requires the result of the Derivation algorithm if $\mathcal{G}_{l} \neq \mathcal{G}_{0}$. It outputs a key $s k_{i}^{l}$ for member $M_{i}$ for the group signature of $\mathcal{G}_{l}$ and the associated revocation token $r t_{i}^{l}$.

The Derivation algorithm is a key step of our setting. A member $M_{i}$ applying to a group $\mathcal{G}_{l}$, child of $\mathcal{G}_{k}$ in $\mathbb{G}$, interacts with the manager $G M_{l}$ of $\mathcal{G}_{l}$. 
$M_{i}$ proves that he owns keys for $\mathcal{G}_{k}$ and, if the proof has been successful, the interaction enables $\mathcal{G}_{l}$ to derive a key for $M_{i}$ for $\mathcal{G}_{l}$ that depends on his key for $\mathcal{G}_{k}$ (but without learning the latter key). Using the thus derived key, $G M_{l}$ can finalize the Enrolment algorithm and provides $M_{i}$ with his new key.

$\operatorname{Derivation}\left(M_{i}, \mathcal{G}_{k}, \mathcal{G}_{l}\right)$ : For a group $\mathcal{G}_{l}$ such that $k \dashv l$, this algorithm is jointly run by a user $M_{i}$ requiring to get group signature keys for the group $\mathcal{G}_{l}$ and the group manager $G M_{l}$ of $\mathcal{G}_{l}$. The input for $M_{i}$ is his group signature key for the parent group $\mathcal{G}_{k}$ of $\mathcal{G}_{l}$ in $\mathbb{G}$ and the input for $G M_{l}$ is his secret key $m s k^{l}$. It returns a new secret key for $M_{i}$ for $\mathcal{G}_{l}$ if $M_{i}$ successfully proves to $G M_{l}$ that he is a non revoked member of $\mathcal{G}_{k}$.

The Sign and Verify algorithms perform the same functionalities as the $\operatorname{Sign}_{G S}$ and Verify $\mathbf{y}_{G S}$ algorithms for a given group $\mathcal{G}_{l}$, using group signatures without Backward Unlinkability, such as the CL scheme for instance. Notice that there are no time periods and the signatures are consequently independent of the time.

$\operatorname{Sign}\left(M_{i}, m, \mathcal{G}_{l}\right)$ : For a group $\mathcal{G}_{l}$, this is run by a user $M_{i}$. The input of $M_{i}$ is his secret $s k_{i}^{l}$ and a message $m$. It returns a group signature $\sigma$ issued by $M_{i}$ on behalf of the group $\mathcal{G}_{l}$.

$\operatorname{Verify}\left(\sigma, m, \mathcal{G}_{l}\right)$ : For a group $\mathcal{G}_{l}$, this is run by anyone knowing $g p k$ and $m p k^{l}$. The inputs are a signature $\sigma$ and a message $m$. It checks if $\sigma$ is a legitimate signature of $m$ by an unrevoked member of $\mathcal{G}_{l}$.

The Revocation algorithm answers to what we expect from our cascade revocation capability. The goal of the downwards revocation process it to ensure that once a user has been revoked from a given group $\mathcal{G}_{l}$, this user is also revoked from all groups that are children of $\mathcal{G}_{l}$ in $\mathbb{G}$, the children of these children, and so on. The optional upwards revocation is there to give the possibility for a group manager to report to the parent group manager that a user has been revoked. If this is not executed, $G M_{k}$ does not learn anything on the identity of the user revoked by $G M_{l}$.

Revocation $\left(M_{i}, \mathcal{G}_{l}\right)$ : This recursive algorithm is run by the group manager $G M_{l}$ of $\mathcal{G}_{l}$ who wants to revoke a member $M_{i}$ of $\mathcal{G}_{l}$. It takes as input the revocation token $r t_{i}^{l}$ of the user $M_{i}$ and the revocation list $R L_{l}$ of $\mathcal{G}_{l}$.

1. Local Revocation: It returns an updated $R L_{l}$ where the revocation token $r t_{i}^{l}$ of $M_{i}$ for $\mathcal{G}_{l}$ has been added.

2. Downwards Revocation (compulsory): The newly published revocation token $r t_{i}^{l}$ is sent to the $G M$ 's of the groups $\mathcal{G}_{m}$ that are children of $\mathcal{G}_{l}$, that then run the Revocation $\left(M_{i}, \mathcal{G}_{m}\right)$ algorithm, after a computation enabling them to retrieve $r t_{i}^{m}$ from $r t_{i}^{l}$.

3. Upwards Revocation (optional): $G M_{l}$ sends an information $r t_{i}^{k \dashv l}$ to the $G M_{k}$ of the group $\mathcal{G}_{k}$ that is the parent of $\mathcal{G}_{l}$, who can then decide to revoke (in that case we will say that the upwards revocation has been accepted) or not the user, using $r t_{i}^{k-l}$ to retrieve the user's revocation token $r t_{i}^{k}$ for $\mathcal{G}_{k}$. 


\subsection{Requirements}

We here describe the security properties that we expect from cross-unlinkable group signatures. Correctness is the same property as in the mono-group setting. Traceability and Cross-Unlinkability are adaptations of Traceability and SelflessAnonymity to our hierarchical group signature setting, with privacy issues in mind.

Correctness: The signature of a member $M_{i}$ who is registered to the group $\mathcal{G}_{l}$ and who is not revoked from this group, using the Sign algorithm is accepted by any verifier that follows the protocol.

Traceability ensures that if a signature $\sigma$ for the group $\mathcal{G}_{l}$ is checked as valid, then the manager $G M_{l}$ of $\mathcal{G}_{l}$ is able to find who made $\sigma$, and it is impossible to mislead him.

Traceability: Setup: The challenger $\mathbf{C}$ runs the KeyGen algorithm, playing the role of all the GM's. He obtains $g p k$ and $\left(m p k^{l}, m s k^{l}\right)$, for each $\mathcal{G}_{l}$. He provides $\mathbf{A}$ with all the $m p k^{l}$ 's and $g p k$.

Queries: A can make the following queries to the challenger:

- Enrol to $\mathcal{G}_{0}$ : A requests the enrolment of a member $M_{i}$ to $\mathcal{G}_{0}$. C obtains the keys for $M_{i}$ for $\mathcal{G}_{0}$. A obtains nothing.

- Derivation: A requests the enrolment of a member $M_{i}$ to $\mathcal{G}_{l}$, provided that $M_{i}$ is already registered to the parent group $\mathcal{G}_{k}$ of $\mathcal{G}_{l}$. $\mathbf{C}$ obtains the keys for $M_{i}$ for the group $\mathcal{G}_{l}$. A obtains the keys only if $M_{i}$ is corrupted. A also obtains all the informations that were exchanged on public channels during the derivation process.

- Sign: A requests that a member $M_{i}$ of a group $\mathcal{G}_{l}$ signs for $\mathcal{G}_{l}$ using a chosen challenge message $m$. The challenger computes the signature $\sigma$ of $M_{i}$ on $m$. A obtains $\sigma$.

- User Corruption: A requests the corruption of a given member $M_{i}$. He obtains all the secret keys of $M_{i}$ for all the groups to which he is registered. $M_{i}$ is revoked from $\mathcal{G}_{0}$, and, through Downwards Revocation, from every group to which he belongs.

- GM Corruption: A requests the corruption of the manager $G M_{l}$ of a group $\mathcal{G}_{l}$. He obtains the secret key $m s k^{l}$ of $G M_{l}$, and all the informations stored by $G M_{l}$ : enrolment informations and the keys (and consequently the revocation tokens $r t_{i}^{l}$ ) of the members of $\mathcal{G}_{l}$.

- Revocation: A requests the revocation of a user $M_{i}$ from a group $\mathcal{G}_{l}$ (and consequently, from all group under $\mathcal{G}_{l}$ in $\mathbb{G}$, through Downwards Revocation). He learns all the revocation tokens of $M_{i}$ that are disclosed during this phase. He can optionally request upwards revocations and, in this case, he learns the informations sent by $G M_{l}$ to the managers of the parent group. If he wants, he can then do a Revocation request on this user for this parent group.

Output: A outputs a challenge message $m$, a signature $\sigma$ and a group $\mathcal{G}_{l}$ such that $G M_{l}$ has not been corrupted. A wins the game if: 
1. $\operatorname{Verify}\left(\sigma, m, \mathcal{G}_{l}\right)=$ valid;

2. A did not obtain $\sigma$ by making a signing query on $m$.

The scheme is said to satisfy Traceability if no polynomial probabilistic adversary is able to win the above game with a non-negligible probability.

The Cross-Unlinkability property is an extension of the Selfless-Anonymity property to the hierarchical group setting. It is not an extension of the Backward Unlinkability property, since we only use one period in the Sign algorithm.

The CU property ensures that a signature issued for the group $\mathcal{G}_{l}$ remains anonymous even for the GM's of other groups, for instance the parent or the sibling groups in $\mathbb{G}$.

We also insist on the fact that, in case of a revocation, if $G M_{l}$ does not inform the manager $G M_{k}$ of the parent group $\mathcal{G}_{k}$ of $\mathcal{G}_{l}$ that a given user is revoked from $\mathcal{G}_{l}$, the manager of $\mathcal{G}_{k}$ is not able to know about the identity of this user.

Cross-Unlinkability: Consider the following CU game played by an adversary

A:

Setup: The challenger $\mathbf{C}$ runs the KeyGen algorithm, playing the role of all the GM's. He obtains $g p k$ and $\left(m p k^{l}, m s k^{l}\right)$, for each $\mathcal{G}_{l}$. He provides $\mathbf{A}$ with all the $m p k^{l}$ 's and $g p k$.

Queries: A can make the following queries to the challenger:

- Enrol to $\mathcal{G}_{0}$ : A requests the enrolment of a member $M_{i}$ to $\mathcal{G}_{0}$. $\mathbf{C}$ obtains the keys for $M_{i}$ for $\mathcal{G}_{0}$. A obtains nothing.

- Derivation: A requests the enrolment of a member $M_{i}$ to $\mathcal{G}_{l}$, provided that $M_{i}$ is already registered to the parent group $\mathcal{G}_{k}$ of $\mathcal{G}_{l}$. C obtains the keys of $M_{i}$ for the group $\mathcal{G}_{l}$. A obtains these keys only if $M_{i}$ is corrupted. A also obtains all the informations that were exchanged on public channels during the derivation process.

- Sign: A requests that a member $M_{i}$ of a group $\mathcal{G}_{l}$ signs for $\mathcal{G}_{l}$ using a chosen challenge message $m$. The challenger computes the signature $\sigma$ of $M_{i}$ on $m$. A obtains $\sigma$.

- User Corruption: A requests the corruption of a given member $M_{i}$. He obtains all the secret keys of $M_{i}$ for all the groups to which he is registered.

- GM Corruption: A requests the corruption of the manager $G M_{l}$ of a group $\mathcal{G}_{l}$. He obtains the secret key $m s k^{l}$ of $G M_{l}$, and all the informations stored by $G M_{l}$ : enrolment informations and the keys (and consequently the revocation tokens $r t_{i}^{l}$ ) of the members of $\mathcal{G}_{l}$.

- Revocation: A requests the revocation of a user $M_{i}$ from a group $\mathcal{G}_{l}$ (and consequently, from all group under $\mathcal{G}_{l}$ in $\mathbb{G}$, through Downwards Revocation). He learns all the revocation tokens of $M_{i}$ that are disclosed during this phase. He can optionally request upwards revocations and, in this case, he learns the informations sent by $G M_{l}$ to the managers of the parent group. If he wants, he can then do a Revocation request on this user for this parent group.

Challenge: A outputs two challenge messages $m$ and $m^{\prime}$, two different members $M_{0}$ and $M_{1}$ and two groups $\mathcal{G}_{k}$ and $\mathcal{G}_{l}$. They must be such that: 
1. $M_{0}$ and $M_{1}$ are registered to both $\mathcal{G}_{k}$ and $\mathcal{G}_{l}$;

2. A corrupted neither $M_{0}$ nor $M_{1}$;

3. A corrupted at most one among $G M_{k}$ and $G M_{l}$. And none is corrupted if $\mathcal{G}_{k}=\mathcal{G}_{l}$;

4. $M_{0}$ and $M_{1}$ are revoked from at most one (and the same for both) group, and, if this is the case, the GM of the other group is not corrupted. If $\mathcal{G}_{k}=\mathcal{G}_{l}$, neither $M_{0}$ nor $M_{1}$ is revoked from $\mathcal{G}_{k}$.

This implies in particular that in the case where $M_{0}$ or $M_{1}$ is revoked from $\mathcal{G}_{l}$ (resp. $\mathcal{G}_{k}$ ) and if $\mathcal{G}_{k}$ is the parent of $\mathcal{G}_{l}$ (resp. $l \dashv k$ ), upwards revocation should not be executed and accepted by the parent group manager.

$\mathbf{C}$ chooses two random bits $b, b^{\prime} \in_{R}\{0,1\}$ and signs for $M_{b}$ on behalf of $\mathcal{G}_{k}$ using message $m$ and for $M_{b^{\prime}}$ on behalf of $\mathcal{G}_{l}$ using message $m^{\prime}$. The respective signatures $\sigma^{*}$ and $\sigma^{*}$ are transmitted to $\mathbf{A}$

Restricted Queries: A can make the same queries as in the Queries phase, as long as he does not contradict the above requirements 1 to 4 of the Challenge phase.

Output: A outputs a guess $\beta^{\prime} \in\{0,1\}$ on the boolean $\beta=\left(b==b^{\prime}\right)$.

The scheme is said to satisfy Cross-Unlinkability if the probability $\mid \operatorname{Pr}(\beta=$ $\left.\beta^{\prime}\right)-1 / 2 \mid$ is negligible.

We can also define a multi-group version of Exculpability, as a straightforward transposition of the definition of Exculpability for VLR group signatures (see $[8,10])$ to our hierarchical setting.

\section{A Construction of Hierarchical Cross-Unlinkable Group Signatures}

In this section we describe our proposal for cross-unlinkable group signatures that follows the model described in the previous section and fulfils the required properties. This proposal is presented using the CL and BP schemes [8] but could use any VLR group signature satisfying Backward Unlinkability such as $[15-17,19]$. We also give the proofs that our construction meets the security requirements described in Section 3.2.

\subsection{The Protocol}

We recall that groups are organized as a tree $\mathbb{G}$ with a root $\mathcal{G}_{0}$. We use the BP construction with the requirement that each group signature linked to a group $\mathcal{G}_{k}$ to have one period per child $\mathcal{G}_{l}$ of $\mathcal{G}_{k}$ in $\mathbb{G}$, called the " $k \dashv l$ " period, that will be used in the Derivation process, but never in the Sign algorithm, where we use a CL signature with the same parameters.

$\operatorname{KeyGen}(\lambda) G M_{0}$ runs the $\operatorname{KeyGen}_{C L}$ algorithm to generate the public parameters $g p k^{0}=g p k$. Then each $G M_{l}$, including $G M_{0}$, creates a CL/BP group key pair $\left(m p k^{l}, m s k^{l}\right)$ using the same group parameters $g p k^{l}=g p k$. 
The $m s k^{l}$ 's are kept secret by the GM's. $g p k$ and all the $m p k^{l}$ 's are published. Moreover, all $G M_{l}$ 's agree on a random choice of period tokens. In every group $\mathcal{G}_{k}$, one token $h_{k \dashv l}$ per child $\mathcal{G}_{l}$ is required for the " $k \dashv l$ " periods. All theses tokens are made public.

For the Enrolment phase, we assume that $M_{i}$ has fulfilled all the conditions to acquire a key for $\mathcal{G}_{l}$. We distinguish two cases of enrolments, either a first enrolment to a group of $\mathbb{G}$, or an enrolment taking place after a derivation process.

$\operatorname{Enrolment}\left(M_{i}, \mathcal{G}_{l}\right) \quad M_{i}$ and $G M_{l}$ jointly run the $\operatorname{Join}_{B P}\left(M_{i}, m s k^{l}, g p k, m p k^{l}\right)$ algorithm of the BP group signature of $\mathcal{G}_{l}$, following two cases:

- Enrolment to $\mathcal{G}_{0}$ : GM and $M_{i}$ follow the BP protocol, in particular $x_{i}^{0}$ is randomly chosen by GM. It outputs a secret key $s k_{i}^{0}=\left(f_{i}^{0}, x_{i}^{0}, A_{i}^{0}\right)$, a global revocation token $r t_{i}^{0}=x_{i}^{0}$ and period revocation tokens $r t_{i}^{0 \dashv m}=$ $\left(h_{0 \dashv m}\right)^{x_{i}^{0}}$, for each child $\mathcal{G}_{m}$ of $\mathcal{G}_{0}$ in $\mathbb{G}$.

- Enrolment after a Derivation: GM uses the output of the Derivation as the choice for $x_{i}^{l}$. It outputs a secret key $s k_{i}^{l}=\left(f_{i}^{l}, x_{i}^{l}, A_{i}^{l}\right)$, a global revocation token $r t_{i}^{l}=x_{i}^{l}$ and period revocation tokens $r t_{i}^{l-t m}=\left(h_{l \dashv m}\right)^{x_{i}^{l}}$, for each child $\mathcal{G}_{m}$ of $\mathcal{G}_{l}$ in $\mathbb{G}$.

At the end of this algorithm, $G M_{l}$ stores $x_{i}^{l}, A_{i}^{l}, r t_{i}^{l}$ and the period revocation tokens. $M_{i}$ gets $s k_{i}^{l}=\left(f_{i}^{l}, x_{i}^{l}, A_{i}^{l}\right)$. This phase is partially done in a secure way so that no eavesdropper can learn the keys $\left(x_{i}^{l}, A_{i}^{l}\right)$ that are sent by $G M_{l}$ to $M_{i}$.

We now explain how to derive signing keys. Let $\mathcal{G}_{k}$ be the parent group of $\mathcal{G}_{l}$ in $\mathbb{G}$ and let us assume that a user $M_{i}$ owns keys for $\mathcal{G}_{k}$ and wants to acquire keys for the group $\mathcal{G}_{l} . M_{i}$ has to engage a specific authentication process with the group manager $G M_{l}$ of $\mathcal{G}_{l}$.

First, the user authenticates to $G M_{l}$ by signing on behalf of $\mathcal{G}_{k}$, parent of $\mathcal{G}_{l}$ in $\mathbb{G}$, to prove that he is allowed to join $\mathcal{G}_{l}$. This signature is associated to the period " $k \dashv l$ ", dedicated to the derivation from $\mathcal{G}_{k}$ to $\mathcal{G}_{l}$. In addition, $M_{i}$ sends his revocation token $r t_{i}^{k \dashv l}$ associated to the " $k \dashv l$ " period.

The group manager $G M_{l}$ then acts as a verifier for the group signature of $\mathcal{G}_{l}$ and first checks the validity of the signature and the fact that $M_{i}$ is not revoked by executing Verify ${ }_{C L}$ (see Section 2.3) on the signature, using the revocation list $R L_{k}$ containing the global revocation tokens of all the revoked members of $\mathcal{G}_{k}$. He then checks that the revocation token $r t_{i}^{k-l l}$ is the one associated to $M_{i}$ for the period " $k \dashv l$ " by executing Verify $\mathbf{y}_{B P}$ on the signature using a period revocation list set as $\left\{r t_{i}^{k \dashv l}\right\}$; if the Revocation Check fails, then $r t_{i}^{k \dashv l}$ is valid.

Then, a key derivation is executed in such a way that the expected revocation mechanisms would apply while satisfying the requirements defined in Section 3.2. We are in fact exploiting the BU property, without introducing periods that really represent time. We only use the $\mathrm{BU}$ part to the purpose of Cross-Unlinkability while ensuring the possibility of later downwards and upwards revocations. Indeed, the group $\mathcal{G}_{l}$ will be in a sense seen as a time period for the group signature associated to $\mathcal{G}_{k}$. 
Once $G M_{l}$ is sure that $M_{i}$ is an unrevoked member of $\mathcal{G}_{k}, M_{i}$ and $G M_{l}$ can run the enrolment procedure for the group $\mathcal{G}_{l}$ with one requirement: $x_{i}^{l}$ is derived from $r t_{i}^{k-l}$ using a hash function and the secret key $m s k^{l}$ of $G M_{l}$ : $x_{i}^{l}=\operatorname{Hash}\left(m s k^{l} \| r t_{i}^{k \dashv l}\right)$. This will be used by our cascade revocation process, since $x_{i}^{l}$ is also the revocation token $r t_{i}^{l}$ of $M_{i}$ for the group $\mathcal{G}_{l}$. As said before, this enrolment phase must be partially executed in a secure environment.

This derivation process is described in Figure 2.

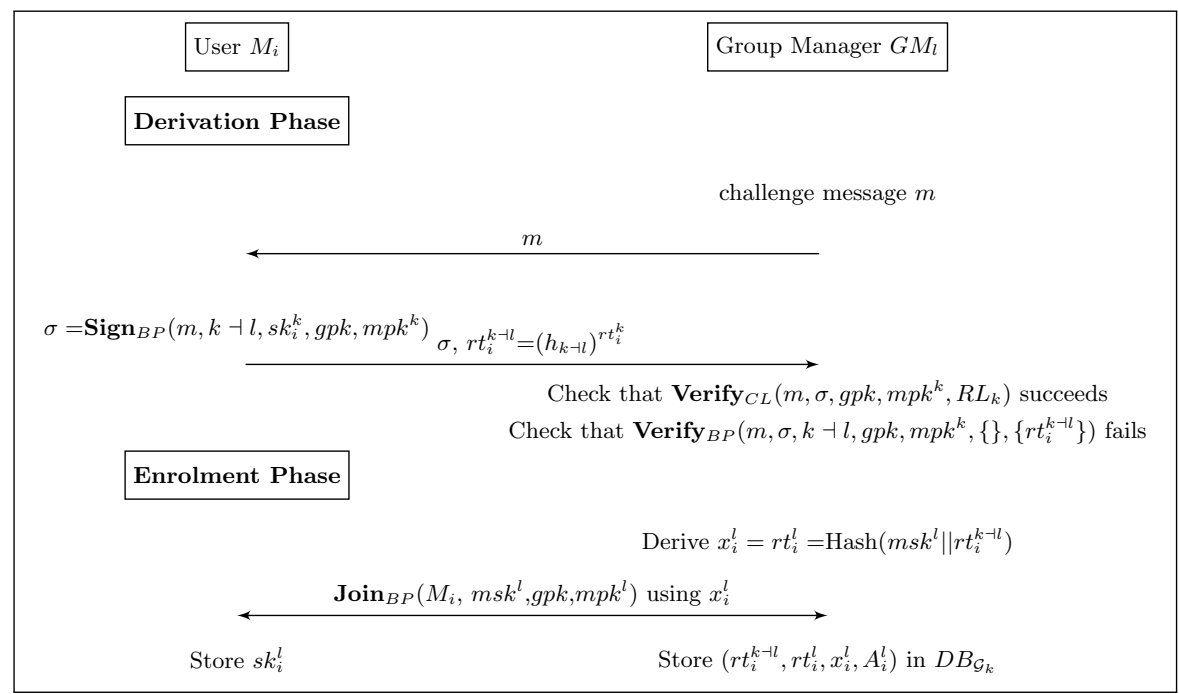

Fig. 2. The derivation process

Derivation $\left(M_{i}, \mathcal{G}_{k}, \mathcal{G}_{l}\right) G M_{l}$ sends a message $m . M_{i}$ signs the challenge message $m$ by executing $\operatorname{Sign}_{B P}\left(m, k \dashv l, s k_{i}^{k}, g p k, m p k^{k}\right)$. He also sends his associated revocation token $r t_{i}^{k \dashv l}$.

$G M_{l}$ checks the signature by executing $\operatorname{Verify}_{C L}\left(m, \sigma, g p k, m p k^{k}, R L_{k}\right)$. In addition, he checks that $r t_{i}^{k-l}$ has been used in the signature, by checking that the revocation check of $\operatorname{Verify}_{B P}\left(m, \sigma, k \dashv l, g p k, m p k^{k},\{\},\left\{r t_{i}^{k \dashv l}\right\}\right)$ fails.

$M_{i}$ and $G M_{l}$ run the Enrolment $\left(M_{i}, \mathcal{G}_{l}\right)$ algorithm using the Enrolment after a Derivation setting with $x_{i}^{l}=\operatorname{Hash}\left(m s k^{l} \| r t_{i}^{k-l}\right)$ as input.

$G M_{l}$ stores in a dedicated database $D B_{\mathcal{G}_{l}}$ the couple $\left(r t_{i}^{k \dashv l}, r t_{i}^{l}\right)$, which will be used for the revocation process.

The Sign and Verify algorithms are direct applications of the CL scheme algorithms. The CL scheme is dedicated to the signature and is not used for derivation. We will also see in the Revocation description that we only use revocation lists made of global revocation tokens. Therefore, we use the Verify ${ }_{C L}$ 
algorithm as Verify algorithm, Verify ${ }_{B P}$ being only used in the derivation process.

$\operatorname{Sign}\left(M_{i}, m, \mathcal{G}_{l}\right) M_{i}$ runs the $\operatorname{Sign}_{C L}$ algorithm of the group signature associated to $\mathcal{G}_{l}$, i.e. he executes $\operatorname{Sign}_{C L}\left(m, s k_{i}^{l}, g p k, m p k^{l}\right)$.

$\operatorname{Verify}\left(\sigma, m, \mathcal{G}_{l}\right)$ The verifier runs $\operatorname{Verify}_{C L}\left(m, \sigma, g p k, m p k^{l}, R L_{l}\right)$.

Let us assume that the group manager $G M_{l}$ of $\mathcal{G}_{l}$ wants to revoke a member $M_{i}$. He proceeds as follows.

Revocation $\left(M_{i}, \mathcal{G}_{l}\right)$ Local Revocation: $G M_{l}$ runs the $\operatorname{Revoke}_{C L}\left(R L_{l}, r t_{i}^{l}\right)$ algorithm. The updated $R L_{l}$ is published.

Downwards Revocation: This direction is automatic. All managers for the children groups $\left(\mathcal{G}_{m}\right)_{m \in M}$ of $\mathcal{G}_{l}$ in $\mathbb{G}$ learn the revocation token $r t_{i}^{l}$. They all compute $\left(h_{l \dashv m}\right)^{r t_{i}^{l}}$ and look in their databases $D B_{\mathcal{G}_{m}}$ 's if this token is present. If it is, they start the $\operatorname{Revocation}\left(M_{i}, \mathcal{G}_{m}\right)$ algorithm for the associated user, using the revocation token $r t_{i}^{m}$ associated to the couple containing $\left(h_{l \dashv m}\right)^{r t_{i}^{l}}$ in $D B_{\mathcal{G}_{m}}$.

Upwards Revocation: We recall that this part of the Revocation algorithm is optional. $G M_{l}$ can report to the manager of the parent group $\mathcal{G}_{k}$ of $\mathcal{G}_{l}$ the user $M_{i}$ if he thinks that $G M_{k}$ should revoke him too. He sends to $G M_{k}$ the item $r t_{i}^{k-l}$ associated to $M_{i}$ in $D B_{\mathcal{G}_{l}}$. If $G M_{k}$ wishes to discover to whom it corresponds, he computes $\left(h_{k \dashv l}\right)^{r t} t_{i^{\prime}}^{k}$ for all the $M_{i^{\prime}}$ 's that belong to $\mathcal{G}_{k}$. When $\left(h_{k \dashv l}\right)^{r t_{i^{\prime}}^{k}}=r t_{i}^{k \dashv l}$, the associated user $M_{i^{\prime}}$ is the user $M_{i}$ that was revoked by $G M_{l}$. $G M_{k}$ can then, if he desires, revoke $M_{i^{\prime}}$ from $\mathcal{G}_{k}$.

Notice that, when $G M_{k}$ does not revoke $M_{i}$, if other GM's, for instance the siblings of $\mathcal{G}_{l}$ in $\mathbb{G}$, have access to the token $r t_{i}^{k-l}$ sent by $G M_{l}$, they cannot link the corresponding member to one of their users, thanks to BU, since $r t_{i}^{k \dashv l}$ is only a period revocation token and thus cannot be linked to the revocation token of the same user for another period.

\subsection{Security Analysis}

We now prove that our construction satisfies the requirements defined in Section 3.2. We rely on the fact that the BP scheme satisfies the properties defined in Section 2.2, i.e. Correctness, Backward-Unlinkability, Exculpability and Traceability, as stated in Theorem 1. Therefore, our construction also relies on the $q$-Strong Diffie-Hellman [4], the adapted Diffie-Hellman [8] and the Discrete Logarithm assumptions.

Theorem 2. The protocol defined in Section 4.1 achieves Correctness.

The proof of Correctness is straightforward, as it only requires that all the underlying BP group signatures achieve Correctness.

Theorem 3. In the random oracle model and under the adapted DDH assumption, the protocol defined in Section 4.1 achieves Cross-Unlinkability. 
Proof (Cross-Unlinkability). Let us assume that there is an adversary $\mathbf{A}$ that is able to win the $\mathrm{CU}$ game with non-negligible probability, we describe how to build an adversary $\mathbf{B}$ that is able to win the BU game of the BP group signature with non negligible probability. Let $\mathcal{G}^{*}$ be the group considered in the BP BU game, the challenger $\mathbf{C}$ considered in the following is the challenger of the $\mathrm{BU}$ game and we use a ${ }^{B U}$ notation to denote a step of the $\mathrm{BU}$ game. We model the hash function Hash as a random oracle. When $\mathbf{B}$ uses it, he picks a uniformly random number while preserving consistency.

$\mathbf{B}$ proceeds as follows to play the BU game with $\mathbf{A}$.

Setup: The challenger $\mathbf{C}$ runs the $\mathbf{K e y G e n}_{C L}$ algorithm, playing the role of the GM $G M^{*}$ of $\mathcal{G}^{*}$. He obtains $g p k^{*}, m p k^{*}$ and $m s k^{*}$. He provides $\mathbf{B}$ with $g p k^{*}$ and $m p k^{*}$. B then builds a group tree $\mathbb{G}$ and picks a random node of this tree to be the one for $\mathcal{G}^{*}$. He fixes the public parameters for $\mathbb{G}$ to be the public parameters $m p k^{*}$ of $\mathcal{G}^{*}$, then chooses secret and public keys for the other groups and provides $\mathbf{A}$ with the public keys.

We assume that the number of period of the group signature of the BU is bigger than the number of children of $\mathcal{G}^{*}$ in $\mathbb{G}$. We use, in addition to the $k \dashv l$ periods, another period called " 0 ".

$\mathbf{B}$ answers to the Queries of $\mathbf{A}$ in the following way:

- Enrol to $\mathcal{G}_{0}$ : If $\mathcal{G}_{0} \neq \mathcal{G}^{*}, \mathbf{B}$ follows the protocol. Otherwise, he makes a $J_{o i n}{ }^{B U}$ request to $\mathbf{C}$ for the considered user $M_{i}$.

- Derivation: When $\mathbf{B}$ requests the enrolment of a member $M_{i}$ to $\mathcal{G}_{l}$, provided that $M_{i}$ is already registered to the parent group $\mathcal{G}_{k}$ of $\mathcal{G}_{l}$, if $\mathcal{G}_{l} \neq \mathcal{G}^{*}$ and $\mathcal{G}_{k} \neq \mathcal{G}^{*}, \mathbf{B}$ follows the protocol.

- If $\mathcal{G}_{k}=\mathcal{G}^{*}, \mathbf{B}$ makes a $\operatorname{Sign}{ }^{B U}$ request to $\mathbf{C}$ on $M_{i}$ for the period " $k \dashv l$ ". He then makes a Revocation ${ }^{B U}$ query on $M_{i}$ for the same period to obtain $r t_{i}^{k \dashv l}$. (This revocation does not impact the sequel, since the user signs at most once for the derivation from $\mathcal{G}_{k}$ to $\mathcal{G}_{l}$. Moreover, thanks to BU, there is no impact on anonymity.) He sends both signature and periodic revocation token to $\mathbf{A}$ and enrols $M_{i}$ to $\mathcal{G}_{l}$ following the protocol.

- If $\mathcal{G}_{l}=\mathcal{G}^{*}, \mathbf{B}$ follows the protocol to issue the signature and the periodic revocation token. He then makes a Join ${ }^{B U}$ request to $\mathbf{C}$ on $M_{i}$. As the hash function used to derive keys is modelled as a random oracle, it is impossible for $\mathbf{A}$ to distinguish between the random choice $x_{i}$ of $\mathbf{C}$ and what should have been really issued with $m s k$ and $r t_{i}^{k-l l}$. Moreover, consistency of the random oracle is preserved with overwhelming probability (it is almost impossible that $\mathbf{B}$ also invokes it on $m s k \| r t_{i}^{k \dashv l}$ ).

- Sign: If $\mathcal{G}_{l} \neq \mathcal{G}^{*}, \mathbf{B}$ follows the protocol. Otherwise, B makes a $\operatorname{Sign}^{B U}$ request to $\mathbf{C}$ for period 0 and member $M_{i}$ and, after having removed the BU part (the items in bold font in the description of Section 2.3), sends the obtained signature $\sigma$ to $\mathbf{A}$.

- User Corruption: $\mathbf{B}$ sends to $\mathbf{A}$ all the secret keys of $M_{i}$ for all the groups $\left(\neq \mathcal{G}^{*}\right)$ to which $M_{i}$ is registered. If $M_{i}$ belongs to $\mathcal{G}^{*}, \mathbf{B}$ also makes a Corruption $^{B U}$ request on $M_{i}$ to $G M^{*}$, obtains the key of $M_{i}$ for $\mathcal{G}^{*}$ and sends it to $\mathbf{A}$. 
- GM Corruption: If $\mathcal{G}_{l} \neq \mathcal{G}^{*}, \mathbf{B}$ follows the protocol. Otherwise, he aborts.

- Revocation: $\mathbf{B}$ follows the protocol as far as $\mathcal{G}^{*}$ is not concerned by the revocation process. If it is, $\mathbf{B}$ requests the global revocation token of the user from $\mathbf{C}$ and publishes it. For the Upwards Revocation case, $\mathbf{B}$ follows the protocol (he is able to do it, since he knows all derivation informations).

Challenge: A outputs two challenge messages $m$ and $m^{\prime}$, two different members $M_{0}$ and $M_{1}$ and two groups $\mathcal{G}_{k}$ and $\mathcal{G}_{l}$ fulfilling the conditions mentioned in the $\mathrm{CU}$ game. If $\mathcal{G}_{k} \neq \mathcal{G}^{*}$ and $\mathcal{G}_{l} \neq \mathcal{G}^{*}, \mathbf{B}$ aborts. Otherwise, we assume w.l.o.g. that $\mathcal{G}_{k}=\mathcal{G}^{*}$. $\mathbf{B}$ chooses a random bit $b^{\prime}$, signs $m^{\prime}$ for $M_{b^{\prime}}$ for the group $\mathcal{G}_{l}$ to obtain $\sigma^{\prime *}$. He then proceeds to the Challenge ${ }^{B U}$ phase with $\mathbf{C}$ with a choice $m, M_{0}, M_{1}$ and period "0". (It is easy to check that we fulfil the requirements of the Challenge ${ }^{B U}$ phase.) $\mathbf{B}$ obtains a signature $\sigma^{*}$. Let us denote by $b$ the random bit chosen by $\mathbf{C} . \sigma^{*}$ and $\sigma^{* *}$ are transmitted to $\mathbf{A}$.

Restricted Queries: A and B interact as in the Queries phase, as long as it does not contradict the requirements of the Challenge phase.

Output: A outputs a guess $\beta^{\prime} \in\{0,1\}$ on the boolean $\beta=\left(b==b^{\prime}\right)$. If $\left(\beta^{\prime}=1\right), \mathbf{B}$ outputs $b^{\prime}$, else it outputs $1-b^{\prime}$.

We can see that the cases of abortion by $\mathbf{B}$ happen with non-overwhelming probability. Since the advantage of $\mathbf{A}$ against the $\mathrm{CU}$ game is non negligible, so is the advantage of $\mathbf{B}$ against the $\mathrm{BU}$ game, which would contradict the fact that the $\mathrm{BP}$ scheme is backward-unlinkable.

Thus, our protocol achieves Cross-Unlinkability.

Theorem 4. In the random oracle model and under the q-SDH assumption, the protocol defined in Section 4.1 achieves Traceability.

Proof (Traceability). Let us assume that there is an adversary $\mathbf{A}$ that is able to win the Traceability game with non-negligible probability, we describe how to build an adversary $\mathbf{B}$ that is able to win the Traceability game of the BP group signature with non negligible probability. Let $\mathcal{G}^{*}$ be the group considered in the BP Traceability game, the challenger $\mathbf{C}$ considered in the following is the challenger of the BP Traceability game. We model the hash function Hash as a random oracle. When $\mathbf{B}$ uses it, he picks a uniformly random number while preserving consistency.

We proceed exactly as in the CU proof for the Setup and the Queries phases. We now explain how to manage the Output phase.

Output: A outputs a message $m$, a signature $\sigma$ and group $\mathcal{G}_{l}$. If $\mathcal{G}_{l} \neq \mathcal{G}^{*}$, abort. Otherwise, $\mathbf{B}$ outputs $m, \sigma$ and period " 0 " to $\mathbf{C}$. Then, as the requirements for both considered games are equivalent, if $\mathbf{A}$ wins the BP Traceability game, $\mathbf{B}$ wins the Traceability game.

We can see that the cases of abortion by $\mathbf{B}$ happen with non-overwhelming probability. Since the advantage of $\mathbf{A}$ against the BP Traceability game is non negligible, so is the advantage of $\mathbf{B}$ against the Traceability game, which would contradict the fact that the $\mathrm{BP}$ scheme is traceable.

Thus, our protocol achieves Traceability. 
The proof that Exculpability of the underlying BP group signature scheme implies Exculpability of the protocol is straightforward.

\section{Application to Anonymous Authentication}

In [7], Bringer et al. suggest to use VLR group signatures to build a biometric anonymous authentication scheme. Their scheme is based on the [5] scheme but can easily be instantiated using any VLR scheme. It can also easily be extended to a non-biometric setting. We describe it with the notations of Section 2.3 for the users keys.

In the [7] setting, members of a group $\mathcal{G}$ authenticate to service providers $P$ (who are different from $G M$ ) while remaining anonymous within $\mathcal{G}$. Moreover, secret keys of the users are derived from an acquisition of a biometric trait. When a user $M_{i}$ applies to join $\mathcal{G}$, an acquisition $b$ of a biometric trait $B$ is made. The group manager and the user then run the $\mathbf{J o i n}_{G S}$ algorithm with the additional requirement that $f_{i}=H(b)$ where $H$ is a hash function. $M_{i}$ then stores $b$ and $x_{i}, A_{i}$ on a device such as a smart-card.

When $M_{i}$ wants to authenticate to a service provider $P$, he connects his device to a trusted sensor. He is acquired a fresh biometric trait $b^{\prime}$. The sensor also gets $b$ and $A_{i}$ from the device. If $b$ and $b^{\prime}$ match, then the sensor signs a challenge message sent by $P$ using $\operatorname{Sign}_{G S}$ with $f_{i}=H(b), x_{i}$ and $A_{i} . P$ then checks the signature using Verify ${ }_{G S}$ and accepts the authentication if the signature is valid. This authentication process is summed up in Figure 3.

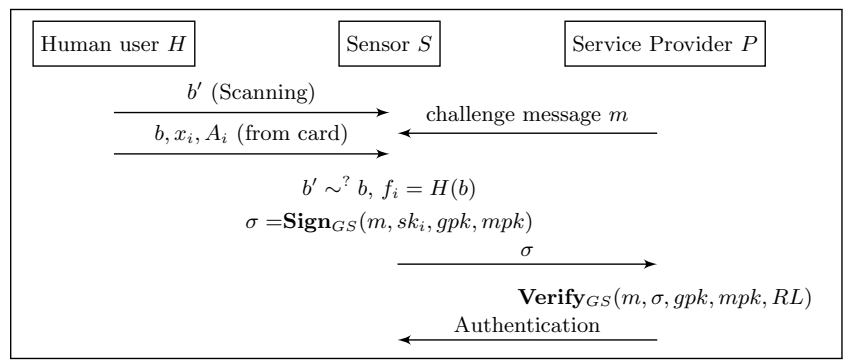

Fig. 3. The [7] authentication scheme.

We can adapt our hierarchical setting to the [7] setting. In this case, several hierarchical groups are available to users and they can anonymously authenticate towards service providers requiring belonging to one or several of these groups. The group signatures associated to these groups are then cross-unlinkable hierarchical group signatures as described in this paper. The adaptation is straightforward and the use of biometrics does not impact our constructions, since we had no requirements on the $f_{i}^{l}$ parts of the secret keys of the users. 
This adaptation of the [7] authentication scheme can for instance be a basis for an identity management system where the groups are the identity domains and where users get identities for these domains, which are not linkable to each other, except in case of revocation.

A more detailed presentation of the adaptation of cross-unlinkable group signatures to biometric identity management can be found in [6].

\section{References}

1. G. Ateniese and G. Tsudik. Some open issues and new directions in group signatures. In Financial Cryptography, pages 196-211, 1999.

2. M. Belenkiy, J. Camenisch, M. Chase, M. Kohlweiss, A. Lysyanskaya, and H. Shacham. Randomizable proofs and delegatable anonymous credentials. In CRYPTO, pages 108-125, 2009.

3. V. Benjumea, S. G. Choi, J. Lopez, and M. Yung. Fair traceable multi-group signatures. In Financial Cryptography, pages 231-246, 2008.

4. D. Boneh and X. Boyen. Short signatures without random oracles. In EUROCRYPT, pages 56-73, 2004.

5. D. Boneh and H. Shacham. Group signatures with verifier-local revocation. In ACM Conference on Computer and Communications Security, pages 168-177, 2004.

6. J. Bringer, H. Chabanne, and A. Patey. An application of a group signature scheme with backward unlinkability to biometric identity management. In SECRYPT, 2012.

7. J. Bringer, H. Chabanne, D. Pointcheval, and S. Zimmer. An application of the Boneh and Shacham group signature scheme to biometric authentication. In IWSEC, pages 219-230, 2008.

8. J. Bringer and A. Patey. VLR group signatures: How to achieve both backward unlinkability and efficient revocation checks. In SECRYPT, 2012. Full version available on the IACR Cryptology ePrint Archive: http: //eprint. iacr .org/2011/376.

9. D. Chaum and E. van Heyst. Group signatures. In EUROCRYPT, pages 257-265, 1991.

10. L. Chen and J. Li. VLR group signatures with indisputable exculpability and efficient revocation. In SocialCom/PASSAT, pages 727-734, 2010.

11. G. Fuchsbauer and D. Pointcheval. Anonymous proxy signatures. In R. Ostrovsky, R. D. Prisco, and I. Visconti, editors, SCN, volume 5229 of Lecture Notes in Computer Science, pages 201-217. Springer, 2008.

12. D. Khader. Attribute based group signature with revocation. IACR Cryptology ePrint Archive, 2007:241, 2007.

13. D. Khader. Attribute based group signatures. IACR Cryptology ePrint Archive, 2007:159, 2007.

14. S. Kim, S. Park, and D. Won. Group signatures for hierarchical multigroups. In ISW, pages 273-281, 1997.

15. B. Libert and D. Vergnaud. Group signatures with verifier-local revocation and backward unlinkability in the standard model. In CANS, pages 498-517, 2009.

16. T. Nakanishi and N. Funabiki. Verifier-local revocation group signature schemes with backward unlinkability from bilinear maps. In ASIACRYPT, pages 533-548, 2005. 
17. T. Nakanishi and N. Funabiki. A short verifier-local revocation group signature scheme with backward unlinkability. In IWSEC, pages 17-32, 2006.

18. M. Trolin and D. Wikström. Hierarchical group signatures. In L. Caires, G. F. Italiano, L. Monteiro, C. Palamidessi, and M. Yung, editors, ICALP, volume 3580 of Lecture Notes in Computer Science, pages 446-458. Springer, 2005.

19. S. Zhou and D. Lin. Shorter verifier-local revocation group signatures from bilinear maps. In $C A N S$, pages 126-143, 2006. 